\title{
Assessment of an Optical Burst Switched Core Node With a Restricted Number of Simultaneous Active Users Using the Burst Error Loss Rate
}

\author{
Ahmed I. Abd El-Rahman, Sherif I. Rabia, and Hossam M. H. Shalaby
}

\begin{abstract}
The technical superiority of spectralamplitude coding optical code-division multiple-access (SAC-OCDMA) systems over traditional wavelength division multiplexing (WDM) systems in optical burst switched (OBS) networks is mainly attributed to the former's better medium access control (MAC) layer performance. Nevertheless, in order to conduct an accurate comparison, a thorough study of the physical layer performance should be involved, especially because in many cases the physical layer noise would affect the maximum achievable number of simultaneously active users. Hence, in this work, we develop a novel assessment approach that combines both MAC and physical layer capabilities by introducing a new burst error loss rate parameter. In particular, the approach targets cases with limitations on the number of simultaneous active users. Next, as an example of a noisy physical layer, the effect of phase-induced intensity noise on the number of active users in OBS/SAC-OCDMA systems is analyzed. Our analysis shows that this effect introduces a burst error rate (BurstER) in the multiple-access interference cancellation operation (not investigated before). This BurstER is an increasing function of the number of active users and hence would suppress the system MAC layer performance. Finally, assuming an ideal WDM physical layer, we employ the developed approach to present an illustrative performance comparison between OBS/SAC-OCDMA and OBS/WDM systems. The results show that OBS/SACOCDMA performance outperforms that of OBS/WDM when the number of tolerated bits in error per burst exceeds a certain value.
\end{abstract}

Index Terms-Optical burst switched (OBS) networks; Phase-induced intensity noise (PIIN); Queuing theory; Spectral-amplitude coding optical code-division multipleaccess (SAC-OCDMA); Wavelength division multiplexing (WDM).

Manuscript received August 27, 2013; revised February 17, 2014; accepted February 17, 2014; published March 31, 2014 (Doc. ID 196098).

A. I. A. El-Rahman (e-mail: ahmed.abdelrahman@queensu.ca) is with the Electrical and Computer Engineering Department, Faculty of Engineering and Applied Science, Queen's University, Kingston, Ontario K7L 3N6, Canada.

S. I. Rabia is with the Department of Engineering Mathematics and Physics, Faculty of Engineering, Alexandria University, Alexandria 21544, Egypt.

H. M. H. Shalaby is with the Department of Electronics and Communication Engineering, Egypt-Japan University of Science and Technology (E-JUST), New Borg El-Arab City, Alexandria 21934, Egypt, and is on leave from the Electrical Engineering Department, Alexandria University, Alexandria 21544, Egypt.

http://dx.doi.org/10.1364/JOCN.6.000408

\section{INTRODUCTION}

$\mathbf{T}$ he ever-growing amount of communicated information has converted the realization of optical Internet to an evident requirement. Among many proposals, optical burst switching (OBS) emerges as a new promising technology on the way to constructing an eminently rapid alloptical network (AON). OBS was first suggested by Qiao and Yoo in [1] as a new solution that effectively uses the vast bandwidth of the optical fiber and makes a huge step forward toward the optical Internet. The key idea behind OBS is to allow the optical data to bypass the optical-toelectronic-to-optical (OEO) conversion. To reach this goal, an OBS network, consisting of three main componentsingress node, core node, and egress node [2] -is built, and a per burst switching process is proposed. At the ingress node, a data burst (DB) is formed by aggregating packets heading to the same destination [3,4]. Next, the burst header, referred to as the control packet $(\mathrm{CP})$, is first transmitted on a separate out-of-band control channel to reserve appropriate resources for its ensuing burst on each core node using a proper reservation protocol $[1,5, \underline{6}]$. The payload waits at the ingress node for an offset time that corresponds to the time required for the header processing operations along the core nodes. Finally, the payload is optically transmitted through the whole way to the egress node, where it is disassembled back to packets.

Researchers have addressed various aspects of OBS networks on many occasions. In particular, factors influencing the contention problem are of major interest. Recently, we have proposed a new CP-buffering-based contention resolution technique [7]. In this technique, an electronic buffer is implemented at each core node. This buffer is employed to save a blocked CP for a predetermined time instead of immediately discarding it. Since the sought resource might be released while its seeking CP is buffered, the blocking probability according to this proposal can be effectively reduced.

A major building block, which strongly contributes to the contention resolution in OBS networks, is the technique by which a user would access the medium, i.e., the resource that the CP seeks to reserve on each core node. Although the traditional wavelength domain has seemed to be the 
first candidate for OBS networks, especially because of the ubiquity of the wavelength routers, Kamakura et al. have suggested to use optical code-division multiple-access (OCDMA) in order to take advantage of the higher system capacity that the code domain can support [8]. In [9], Sowailem et al. have adopted the spectral-amplitude coding CDMA (SAC-OCDMA) technique to present a detailed OBS/ SAC-OCDMA system architecture. Since a simple SACOCDMA encoding/decoding system can be implemented using fiber Bragg gratings (FBGs), they have designed a core node capable of conducting a code-based routing process and have proposed a system that theoretically outperforms traditional wavelength division multiplexing (WDM) systems. For simplicity, only a medium access control (MAC) layer comparison between both systems has been considered. However, in practical cases, physical layer noise represents a principle factor in the comparison. Moreover, in many cases, the dominating noise would affect the MAC layer by restricting the maximum number of simultaneous users. Consequently, there is no guaranteed privilege of a certain medium access technique over the other. Thus, a generalized evaluation strategy that truly expresses the system capabilities in both MAC and physical layers is required. This design tool can be employed to determine, relative to the conditions of each OBS network [e.g., the available number of resources, the maximum sustainable bit-error rate (BER), and the maximum achievable number of simultaneous users], the suitable medium access technique that efficiently fits in this network.

More specifically, in [9], the effect of the interference between incoherent sources, which causes intensity noise, labeled by phase-induced intensity noise (PIIN) $[10,11]$, has not been taken into consideration. The importance of studying such noise is that it exists inherently in OCDMA systems, affects the DB extraction process, and hence puts an upper bound on the allowable number of active users.

Previous research works have analyzed the PIIN in order to find the noise power spectra, noise variance, and covariance under different conditions $[10,12]$. On the other hand, in [13] Smith et al. suggested to use pulseposition modulation (PPM) to reduce the PIIN effect. Another solution was suggested by Wei et al. in [14,15], where they proposed a new code family, namely modified congruence code (MQC) $\left(p^{2}+p, p, 1\right)$. Owing to its lower cross correlation, MQC is more robust against the PIIN than the Hadamard signature code. Moreover, increasing the prime number " $p$ " would improve the system performance even further.

The aim of this paper is to present an exact performance evaluation approach for an OBS system with a limited number of active users. In that regard, a new measuring parameter, namely the burst error loss rate (BELR), is introduced. This parameter refers to the probability that burst is lost (due to contention) or discarded as the number of erroneous bits exceeds a certain threshold. The term BELR has been chosen to be close to the definition and to give a hint about the mathematical model that will be developed. The presented assessment approach, as stated earlier, represents a major step in the network design.
Indeed, burst loss (BL) reflects the MAC layer performance while BER determines the physical layer performance. However, each of these tools gives information about the corresponding layer independently. Therefore, it would be more adequate to express the network performance in terms of a third measure that assesses the overall network performance. In that regard, BELR is a metric for the overall behavior of the system. It could be used to judge proposed system improvement. However, from an analytical point of view, we cannot compute this metric directly. We need to derive both the burst error rate (BurstER) and the overall BL rate to find an expression for BELR. Based on simulation, this metric could be estimated directly, and hence analytical results may be compared to simulation results to test the validity of the analytical model and assist approximations usually assumed during mathematical modeling. Moreover, we investigate the OBS/SAC-OCDMA system physical layer performance when the effect of PIIN is considered, as this type of noise is related to the number of simultaneous users. Finally, we use the developed approach to present a comparison between a noisy physical layer OBS/SAC-OCDMA system with an ideal physical layer OBS/SAC-OCDMA system. Briefly, the novelty of this paper comprises the following: We derive a mathematical model for an OBS network that has a predetermined number of resources, but a restricted number of simultaneous users; i.e., only a fraction of the available resources is allowed to be busy simultaneously. Next, we employ the results obtained in $[10,11,13,14]$ to evaluate the PIIN effect on the OBS/SAC-OCDMA optical layer BER. Furthermore, we point out that the adoption of ideal in-phase crosscorrelation codes is more convenient for OBS/SAC-OCDMA systems than the Hadamard code suggested in [9]. Thus, we adopt the MQC family instead of the Hadamard code in order to effectively suppress the PIIN effect and maintain the higher capacity of OBS/SAC-OCDMA systems over OBS/WDM systems. Indeed, the intention behind adopting such a code family is simply to employ it in OBS/SACOCDMA systems rather than prove their robustness against PIIN, as this has been proved previously in the literature. Finally, we compare between OBS/SAC-OCDMA and OBS/WDM systems using the BELR measure. For simplicity, we assume an ideal WDM physical layer, which gives an extra advantage to WDM over the OCDMA technique. However, the developed comparison approach can be involved in general cases to reach an accurate network design.

The rest of the paper is organized as follows: The mathematical model of the assessment approach is presented in Section II. In Section III, an evaluation of the effect of PIIN on the multiple interference cancellation process in OBS/SAC-OCDMA systems is given. Next, using the performance measures derived in previous sections some numerical results are presented in Section IV. Finally, our conclusions are given in Section V.

\section{Proposed Assessment Approach}

In OBS networks, each link is assigned a fixed number of resources, i.e., wavelengths or codes. Ideal MAC layer 
evaluation processes always assume that the number of available resources on each link can be exhaustively used without any restrictions. Nevertheless, the presence of active user-dependent noise would require a more appropriate treatment of the problem. In such cases, the resulting BER is directly proportional to the number of simultaneous users. Consequently, the allowable number of simultaneous users must be adapted according to the maximum sustainable BER, as otherwise, the system performance would be drastically affected. That is, only a portion of the available resources can be reserved simultaneously. Accordingly, accurate calculation of the per node blocking probability should be performed in a different manner than that in ideal cases. Moreover, the system evaluation process must take the physical layer resulting BER into consideration. The following model proposes a mathematical expression of an overall burst loss or error probability, provided that only a limited number of the available resources can be used simultaneously.

\section{A. MAC Layer Burst Loss Probability}

For simplicity, we study an output link of an isolated core node. First, we start by treating the case in which the evaluated core node does not employ resource converters, i.e., neither code nor wavelength converters are deployed. (Resource converters will be considered later on.) Consequently, an arriving burst seeks to be routed on a specific resource that cannot be changed. Furthermore, exponentially distributed burst interarrival and service times are assumed with average rates $\lambda$ and $\mu$, respectively. Though high-resolution traffic measurement proves that Internet traffic streams follow the self-similarity (long-range dependency, LRD) property, the assumption of Poisson arrivals seems suitable to many cases, where the adopted assembly algorithm smooths the traffic $[\underline{3}, \underline{16}]$.

Finally, we assume that the offered load is uniformly distributed among the available resources. In other words, the probability that an arriving burst targets a specific resource is the same for all resources. For an ideal physical layer, each resource is treated as an independent server and the $M / M / 1 / 1$ queuing model can be safely used. According to the Erlang-B formula the per node BL probability $P_{\text {Node-Loss }}$ (for an ideal physical layer) can be written as [17]

$$
P_{\text {Node-Loss }}=\frac{\rho / N}{1+\rho / N}
$$

where $\rho$ is the offered load, defined as $\rho=\lambda / \mu$, and $N$ is the number of available resources.

Obviously, the last equation describes the loss event that occurs when a burst finds its required resource busy upon arrival. Now, let us assume that only $K$ out of $N$ are allowed to be busy simultaneously. That is, observing any output link of the core node, one may find at most $K$ busy resources [Fig. 1(a)]. Consequently, Eq. (1), due to this restriction, is no longer valid.

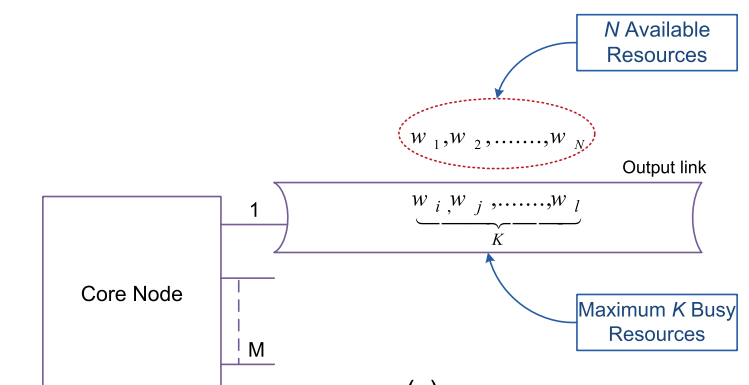

(a)

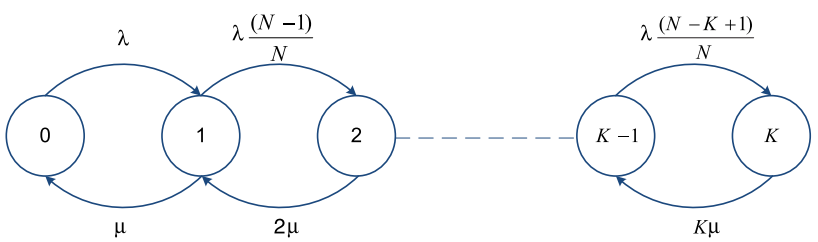

(b)

Fig. 1. (a) Illustration of an output link with limited number of active users. (b) System state diagram.

In order to model such a case, we proceed as follows: First, note that the probability that an arrival seeks a specific resource equals $1 / N$. Next, assuming that a CP finds $i$ busy resources upon arrival, the probability that it finds its sought resource free equals $(N-i) / N$. Furthermore, let $X(t)$ denote the number of busy channels at an arbitrary time $t$. Based on the stated description, $X(t)$ is a continuous time Markov chain (CTMC) with state space $S=\{i: 0 \leq i \leq K\}$. In particular, $X(t)$ is a birth-death process with the following types of transitions:

1) The process makes a forward transition, i.e., from $(i)$ to $(i+1)$, for $0 \leq i \leq K-1$ with a new arrival requiring any free resource. Accordingly, the transition rate is given by $\lambda(N-i) / N$.

2) The process makes a backward transition, i.e., from $(i)$ to $(i-1)$, for $1 \leq i \leq K$ with a new departure, and the transition rate is $i \mu$.

Following these conditions, the state transition diagram is illustrated in Fig. 1(b), and the stationary distribution of $X(t)$ can be easily derived to get

$$
\pi_{k}=\pi_{0} \prod_{i=1}^{k} \frac{\lambda((N-i+1) / N)}{i \mu}=\pi_{0}\left(\frac{\lambda}{N \mu}\right)^{k}\left(\begin{array}{c}
N \\
k
\end{array}\right),
$$

where

$$
\pi_{0}=\left[1+\sum_{k=1}^{K}\left(\frac{\lambda}{N \mu}\right)^{k}\left(\begin{array}{c}
N \\
k
\end{array}\right)\right]^{-1}
$$

The next step is to find the BL probability. Intuitively, a BL will result if the CP arrives when the system is in state $K$ or if it finds the desired channel busy provided that the number of busy channels has not reached $K$ yet. Thus, the BL probability can be obtained as 


$$
P_{\text {Node-Loss }}=\pi_{K}+\sum_{i=1}^{K-1} \pi_{i} \times \frac{i}{N}
$$

Note that Eq. (1) can be obtained from Eq. (3) by removing the restriction of $K$ simultaneous users.

Let us extend the model to comprise cases in which resource converters are implemented to combat the probable contention. Simply, the role of a resource converter is to switch the burst bearing channel, which is currently busy, to an idle one. Either full or partial conversion (FC or PC) can be adopted. In the case of $\mathrm{FC}$, a burst arriving at a certain channel can be converted to any free channel. However, recall that only $K$ channels out of $N$ can be used simultaneously and hence a maximum number of $K$ converters can be deployed per output port. Accordingly, the output port can be modeled as an $M / M / K / K$ loss system, and the loss probability can be obtained as

$$
P_{\text {Node-Loss }}=\frac{\rho^{K} / K !}{\sum_{i=0}^{K} \rho^{i} / i}
$$

Since the FC strategy is costly, PC (converter sharing) is more common. There exist several architectures for converter sharing. In this work, we follow the share-per-line (SPL) architecture adopted in [18]. In such architecture, a number of resource converters $C$ (where $0<C<K$ ) is implemented on each output port to be shared among the DBs arriving to this port. As described in [18], this case is analyzed using a two-dimensional CTMC $X(t)=\{i(t), j(t)\}$, where $i(t)$ and $j(t)$ refer to the number of busy channels and the number of busy converters at an arbitrary time $t$, respectively. Unlike [9] and [18], the system state space can be written as $\overline{S=}\{(i, k): 0 \leq i \leq K, 0 \leq j \leq \min (i, C)\}$, and hence the transition rate matrix $Q$ is truncated to cover the states $\{(0,0), \ldots,(K, C)\}$ only. Furthermore, assuming that the system currently lies in state $(i, j)$, we briefly state the events that lead to a state transition:

\section{- Forward transitions:}

- The system jumps to state $(i+1, j)$, for $0 \leq i \leq K-1$ and $0 \leq j \leq \min (i, C)$, when an arriving DB targets a free resource with rate $\lambda(N-i) / N$.

- The system jumps to state $(i+1, j+1)$, for $0 \leq i \leq K-$ 1 and $0 \leq j \leq \min (i, C-1)$, when an arriving DB targets a busy but convertible resource (as there still exist free converters) with rate $i \lambda / N$.

\section{- Backward transitions:}

- The system jumps to $(i-1, j)$, for $1 \leq i \leq K$ and $0 \leq j \leq \min (i, C)$, with a departing DB that was not using a converter with rate $\mu(i-j)$.

- The system jumps to $(i-1, j-1)$, for $1 \leq i \leq K$ and $1 \leq j \leq \min (i, C)$, with a departing DB that was using a converter with rate $j \mu$.

Using these rates, the long run state probabilities can be deduced by decomposing the state space into levels based on the number of busy resources $i(t)$ and applying the block tridiagonal LU factorization algorithm described in [18]. Finally, the blocking probability can be written as

$$
P_{\text {Node-Loss }}=\sum_{j=0}^{C} \pi_{K, j}+\sum_{i=C}^{K-1} \pi_{i, C} \times \frac{i}{N}
$$

\section{B. Burst Error Loss Rate}

The approach we use to evaluate the performance of the system is to find its BL or error probability. The BL is given by Eq. (1) (ideal case) and Eqs. (3), (4), and (5) (practical case). Thus, it is now required to calculate the burst error probability. Let $l_{e}$ be the maximum tolerated number of bits in error out of $l_{B}$, where $l_{B}$ is the burst length. That is, a burst is considered erroneous when it contains a number of bits in error $>l_{e}$. Moreover, let BER denote the bit-error rate. Thus, assuming that we have $H$ hops, i.e., $H$ core nodes along the way from ingress node to egress node, the overall $\mathrm{BER}_{H}$ can be found as

$$
\mathrm{BER}_{H}=1-(1-\mathrm{BER})^{H},
$$

and the burst error probability can be written using the binomial distribution as follows:

$$
\operatorname{BurstER}\left(l_{B}\right)=\sum_{i=l_{e}+1}^{l_{B}}\left(\begin{array}{c}
l_{B} \\
i
\end{array}\right)\left(\mathrm{BER}_{H}\right)^{i}\left(1-\mathrm{BER}_{H}\right)^{l_{B}-i}
$$

Note that the smallest burst bears one packet and the minimum packet size is several tens of bytes (e.g., 48, 64 bytes). That is, the burst length $l_{B}$ is at least several hundreds of bits. Hence, assuming that the minimum burst length $l_{B}=$ 100 bits and given that the value of the BER is normally small, we can employ the Poisson approximation of the binomial distribution [19]:

$$
\operatorname{BurstER}\left(l_{B}\right)=e^{-\left(l_{B} \cdot \mathrm{BER}_{H}\right)} \sum_{i=l_{e}+1}^{l_{B}} \frac{\left(l_{B} \cdot \mathrm{BER}_{H}\right)^{i}}{i !} .
$$

Needless to say, the burst length is also an exponentially distributed random variable $\left(L_{B}\right)$ as it is related to service time by $1 / R_{b}$, where $R_{b}$ is the nominal bit rate. Nevertheless, as assumed earlier, $L_{B}$ is defined in the interval $100 \leq l_{B}<\infty$. Hence, a truncated exponential distribution must be involved. However, the remarkable memory-less property of the exponential distribution states that the new density function is an exact replica of the original function, except for a right shift [20]. That is, $L_{B}$ has the following probability density function (PDF):

$$
P_{L_{B}}\left(l_{B}\right)=\frac{\mu}{R_{b}} e^{-\frac{\mu}{R_{b}}\left(l_{B}-100\right)}
$$

Consequently, the overall BurstER can be found by employing the total probability theorem as follows: 


$$
\begin{aligned}
\text { BurstER }= & \int_{l_{B}=100}^{\infty} e^{-\left(l_{B} \cdot \mathrm{BER}_{H}\right)} \sum_{i=l_{e}+1}^{l_{B}} \frac{\left(l_{B} \cdot \mathrm{BER}_{H}\right)^{i}}{i !} \\
& \times \frac{\mu}{R_{b}} e^{-\frac{\mu}{R_{b}}\left(l_{B}-100\right)} \mathrm{d} l_{B} .
\end{aligned}
$$

In fact, finding a closed-form solution to the last equation is nontrivial. Hence, we may substitute the parameters $\mu$, BER, and $R_{b}$ by explicit values and perform the calculations numerically using MATLAB. The last step is to combine the BurstER and the overall $B L$ rate $\mathrm{BL}_{H}$ to find the BELR as follows: A successful burst transmission in burst switching strategy implies the success of the CP in each core node along the burst path (MAC layer success), then a correct transmission of burst bits. Thus, assuming that the burst path contains $H$ hops, the BELR is given by

$$
\mathrm{BELR}=1-\left(1-\mathrm{BL}_{H}\right)(1-\mathrm{BurstER},
$$

where the $\mathrm{BL}_{H}$ is given by

$$
\mathrm{BL}_{H}=1-\left(1-P_{\text {Node-Loss }}\right)^{H} .
$$

\section{Analysis of a PRActical OBS/SAC-OCDMA SYSTEM}

Next, we exemplify a system that suffers from active user-dependent noise by analyzing the effect of PIIN on the OBS/SAC-OCDMA proposed in [9].

\section{A. Preliminaries}

1) Multiple-Access Interference Cancellation Process: Since the forwarding process in each core node is codebased, an all-optical multiple-access interference (MAI) canceler, shown in Fig. 2, was suggested to extract the DB on a distinct code from its interferers [9]. Furthermore, recall that the system employs on-off keying (OOK) instead of the complementary coding to reduce the system complexity; i.e., the encoder emits a signal power when data bit " 1 " is sent, while it produces no power when data bit " 0 " is sent. Therefore, in this MAI canceler the OOK encoded bits of the burst pass, bit by bit, through FBG1, which is tuned to the desired code to separate the desired spectral slices.

The FBG is a piece of fiber that is fabricated such that a periodic change in the refraction index is introduced along the fiber axis. This design allows the grating to act as a wavelength selective filter; i.e., it rejects only wavelengths that satisfy the Bragg condition. In tunable FBGs, these wavelengths can be controlled by controlling either Bragg temperature or strain.

Based on the correlation property of the Hadamard codes, which implies that an interfering code overlaps with the desired code and its complement in exactly $L / 4$ mark positions, where $L$ is the code length, the output power $P_{\text {upper }}$ of FBG1 represents the contribution of the desired

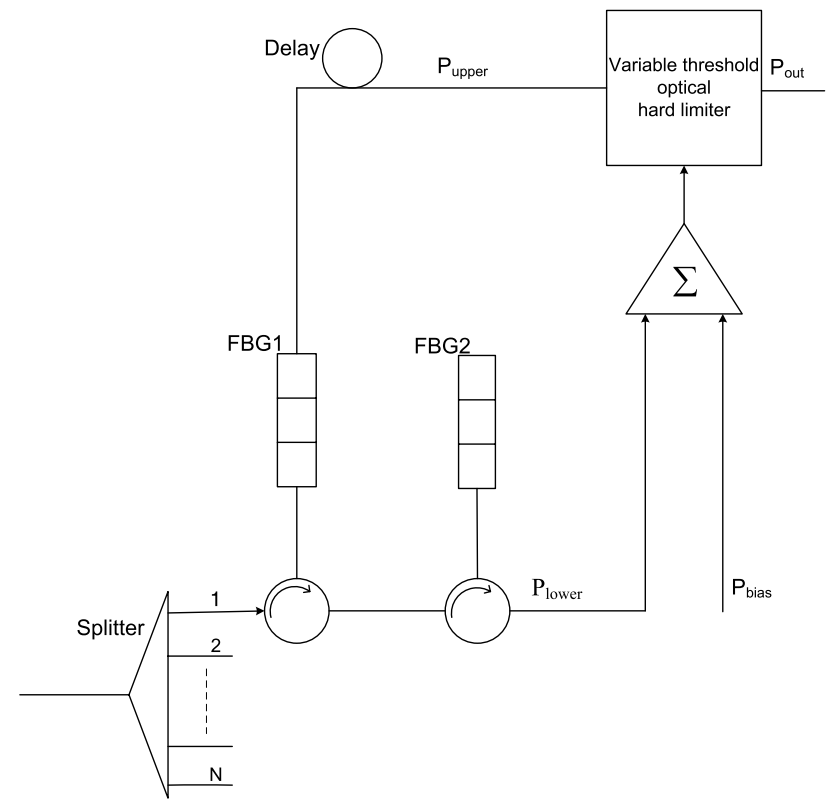

Fig. 2. All-optical multiple-access interference canceler.

DB slices as well as the overlapping slices belonging to interferers. On the other hand, the reflected power is the contribution of the interferers in the complement parts of the spectrum. That is why this upper branch output is next applied to an optical variable threshold hard limiter, while the lower branch output is used to control the hard limiter threshold power $P_{\text {th }}$. Recall that, although there is an analogy between the functions performed by both devices, a balanced detector cannot replace the variable threshold optical hard limiter in the core nodes. This can be justified as follows: The key idea in OBS networks is to separate the $\mathrm{CP}$ from the DB, so that the DB can be optically routed through the whole network. Thus, any required processing for the DB along its path through the core nodes must be performed in the optical domain. Consequently, the authors of [9] suggested using an MAI canceler with a variable threshold optical hard limiter in order to extract the desired DB from the interferers in the optical domain. Briefly, a simple balanced detector, which eventually converts the signal from the optical domain to the electronic domain, can be used at the egress node but not in the core nodes.

In this paper we propose to replace the Hadamard code by the $\left(p^{2}+p, p+1,1\right)$ modified quadratic congruence code series, developed by Wei et al. [14,15]. The MQC is characterized by a fixed in-phase cross correlation that is always equal to 1 . This property is the key behind the MQC code ability to suppress the PIIN. Furthermore, the authors designed an encoder/decoder composed of FBGs. Thus, the MQC can be employed in OBS/SACOCDMA systems instead of the Hadamard code with the same core node structure suggested in [9]. Only a slight modification must be performed to the $\bar{M}$ AI canceler by adding a $1 / p$ divider. In addition, a different threshold decision level must be introduced to accommodate the system for the MQC properties, as we will show in the analysis. 
2) Phase-Induced Intensity Noise: For simplicity, we assume that $P_{r}$ represents the average power received from each source after the splitter. Hence any excess noise is assumed to be incorporated in $P_{r}$. In addition, we assume that the MAI canceler is ideal. Thus, a decision about the sent bit is made as follows:

$P_{\text {out }}=P_{r}, \quad$ for $\quad P_{\text {upper }} \geq P_{\text {th }}, P_{\text {out }}=0, \quad$ for $\quad P_{\text {upper }}<P_{\text {th }}$.

In fact, a thorough observation about $P_{\text {upper }}$ and $P_{\text {lower }}$ reveals that they consist of a mix of incoherent light fields. This mixing operation gives rise to fluctuations in the signal intensity, and consequently in the signal power, caused by the phase noise of the fields and called PIIN [21]. However, both branches contain the contributions of nonoverlapping regions of the spectrum. That is, the intensity noise embedded in each branch will be independent of that in the other branch. The effect of this noise is a main factor that must be taken into consideration when analyzing SAC-OCDMA systems, simply because it affects the number of active users and determines the system capacity.

A brief explanation of the PIIN effect is given in the following example: Assume we have two light sources that generate two signals $S_{1}$ and $S_{2}$ of the same frequency but of statistically independent random phases. Next, $S_{1}$ and $S_{2}$ pass through fiber delay lines "FDL ${ }_{1}$ " and "FDL ${ }_{2}$," respectively. Finally, $S_{1}$ and $S_{2}$ are mixed after experiencing different delays. The consequence of this mixing operation is that the intensity of the resultant signal is not deterministic anymore but contains an induced intensity noise caused by the random phase of each signal as stated earlier. This is interpreted by the fact that the intensity represents the square of the added fields. In other words, the nonlinear effect of the squaring operation of two fields of the same frequency, statistically independent random phase, and different delays, yields terms of coherent addition of the fields $E_{1}^{2}+E_{2}^{2}$ and "cross terms" that represent the intensity noise [11]. Thus, the PIIN affects any decisioning operation that depends on the signal intensity regardless of the device that performs this operation.

Now we apply this argument to our case in which an optical hard limiter is employed as a decision device. The main idea of the optical hard limiter is that it limits the signal intensity to zero or to a certain level based on the threshold value. Theoretically, it senses the signal intensity and senses the threshold intensity and then compares them and finally reaches the decision. Taking into consideration the effect of the PIIN, an error might occur in the sensing phase leading to a wrong decision.

In [10] a closed form for this PIIN power spectral density (PSD) was derived for both coherent and incoherent regimes. In the case studied here, we are concerned only with the incoherent regime, where the optical band of operation is much larger than the information bandwidth. That is, the bit duration $T \gg$ source coherence time $\tau_{c}$. It should be noted that since the signal noise is in principle related to the source temporal coherence and since the desired signal is applied to the surface of the hard limiter for a time $T$, the amount of noise power, i.e., noise variance, that affects the system is a function of the carried information bandwidth $B$ and the source coherence time $\tau_{c}$. Hence, for two uncorrelated, unpolarized sources, the intensity noise variance can be approximated as follows $[\underline{10}, \underline{13}]$ :

$$
\sigma_{P}^{2} \cong \frac{1}{2} P^{2} \tau_{c} B,
$$

and the signal mean is given by

$$
\text { mean } \cong P,
$$

where $P$ is the source average power, i.e., the power resulting from the fields' intensity coherent sum. As discussed in [13], let us assume that the number of users is large enough to allow the PIIN to dominate over noise originating from individual sources. Inspired by this assumption, we can proceed to find an expression for the noise variance, assuming that the output signals of the upper and lower branches act as if they were originated from a single source with a PSD that equals the sum of PSDs of each user $[\underline{13}, 22]$. This will become clear when calculating $\tau_{c}$ below.

\section{B. MAI Canceler Performance Analysis}

1) Noise Variance Calculations: First consider the following list of variables with their definitions:

- $c_{m}(i), c_{m}^{-}(i)$ represent the $i$ th element of the $m$ th signature code sequence and the $i$ th element of its complement, respectively. On the other hand, $c_{m}$ and $c_{m}^{-}$refer to the $m$ th code sequence and its complement, respectively. That is, $c_{m}=\left\{c_{m}(1), c_{m}(2), \ldots, c_{m}(L)\right\}$, where $L$ is the code length.

- $P_{r}$ is the received power from a single source. Assuming that each unpolarized source emits a flat PSD over a band $\Delta f$, the magnitude of the source PSD will be $P_{r} / \Delta f$. It should be noted that $\Delta f=\Delta \lambda c / \lambda_{0}^{2}$, where $\Delta \lambda$ is the spectral width in nanometers, $\lambda_{0}$ is the operating wavelength, and $c$ is the speed of light.

- $S_{\text {upper } \mid b}(f)$ represents the PSD of the upper branch given that the desired user is sending " $b$," where $b \in\{0,1\}$. In the following analysis we assume, without loss of generality, that FBG1 is tuned to retrieve $c_{1}$. That is, $S_{\text {upper|1 }}(f)$ will be the spectrum slices corresponding to the mark positions in $c_{1}$.

- $S_{\text {lower }}(f)$ denotes the PSD of the lower branch. On the contrary to $S_{\text {upper|b}}(f), S_{\text {lower }}(f)$ represents the spectrum slices corresponding to $c_{1}^{-}$.

- $k \in\{0,1,2, \ldots, K\}$ denotes the number of interfering users sending " 1 " out of $K$ interfering users. In other words, we have $K$ interfering users with the following distribution: $K-k$ users are sending " 0 ," and $k$ users are sending "1." In addition, we have one desired user sending " 1 " or " 0 ." Hence, the total number of active users is $K+1$.

As stated earlier, in the MQC case, the overlap of an interferer with the desired code will be in only one position. 
Consequently, the code properties, for any $m, n \in\left\{1,2, \ldots, p^{2}\right\}$, can be written as follows:

$$
\begin{gathered}
\sum_{i=1}^{L} c_{m}(i) c_{n}(i)=\left\{\begin{array}{cc}
p+1, & m=n \\
1, & m \neq n
\end{array}\right. \\
\sum_{i=1}^{L} c_{m}(i) c_{n}^{-}(i)=\left\{\begin{array}{cc}
0, & m=n \\
p, & m \neq n
\end{array}\right.
\end{gathered}
$$

Recall that the code length $L$ is given by

$$
L=p^{2}+p,
$$

and the maximum number of available codes $N=p^{2}$.

Thus, using Eqs. ( $\underline{14 \mathrm{a})}$ and (14b), $P_{\text {upper }}$ and $P_{\text {lower }}$ can be written as follows:

$$
\begin{gathered}
P_{\text {upper } \mid 1}=\frac{P_{r}}{L}(p+k+1), \\
P_{\text {upper } \mid 0}=P_{\text {lower }}=\frac{P_{r} k}{L} .
\end{gathered}
$$

Now, let us start by calculating $\tau_{c}$ :

$$
\tau_{c}=\frac{\int_{0}^{\infty} S^{2}(f) \mathrm{d} f}{\left[\int_{0}^{\infty} S(f) \mathrm{d} f\right]^{2}},
$$

where $S(f)$ is the signal PSD, which is the sum of the PSDs of the desired user and contributions of the interfering users, i.e., users having slices of their spectrum overlapping with the desired spectrum slices. Thus, the upper and lower PSDs can be mathematically expressed as follows:

$$
S(f)_{\mathrm{upper} \mid 1}=\frac{P_{r}}{\Delta f} \sum_{m=1}^{k+1} \sum_{i=1}^{L} c_{m}(i) c_{1}(i) \operatorname{rect}(i),
$$

and

$$
S(f)_{\mathrm{upper} \mid 0}=\frac{P_{r}}{\Delta f} \sum_{m=2}^{k+1} \sum_{i=1}^{L} c_{m}(i) c_{1}(i) \operatorname{rect}(i)
$$

while

$$
S(f)_{\text {lower }}=\frac{P_{r}}{\Delta f p} \sum_{m=2}^{k+1} \sum_{i=1}^{L} c_{m}(i) c_{1}^{-}(i) \operatorname{rect}(i),
$$

where rect $(i)$ can be expressed in terms of the unit step function $u(f)$ as follows [13]:

$$
\begin{aligned}
\operatorname{rect}(i)= & u\left(f-f_{o}-\frac{\Delta f}{2 L}(-L+2 i-2)\right) \\
& -u\left(f-f_{o}-\frac{\Delta f}{2 L}(-L+2 i)\right),
\end{aligned}
$$

and $f_{o}$ is the laser center frequency.
It should be noted that the signal average power can be written as

$$
P=\int_{0}^{\infty} S(f) \mathrm{d} f
$$

Next, plugging the explicit values of $P$ and $\tau_{c}$ into Eq. (12) yields

$$
\sigma_{P}^{2}=\frac{1}{2} B \int_{0}^{\infty} S^{2}(f) \mathrm{d} f
$$

Hence, it is required to calculate the nominator of Eq. (16) only. Employing Eq. (17) we get [1]

$$
\int_{0}^{\infty}\left[S(f)_{\mathrm{upper} \mid 1}\right]^{2} \mathrm{~d} f=\frac{P_{r}^{2}}{L \Delta f} \sum_{i=1}^{L} c_{1}(i) \cdot\left[\sum_{m=1}^{k+1} c_{m}(i)\right] \cdot\left[\sum_{n=1}^{k+1} c_{n}(i)\right] .
$$

Moreover, using the MQC correlation properties, Eq. (22) may be accurately expanded to get [23]

$$
\int_{0}^{\infty}\left[S(f)_{\mathrm{upper} \mid 1}\right]^{2} \mathrm{~d} f=\frac{P_{r}^{2}}{L \Delta f}\left[p+3 k+1+\frac{k(k-1)}{p}\right] .
$$

Next, we use Eqs. (18) and (19) in a similar manner to get

$$
\begin{gathered}
\int_{0}^{\infty}\left[S(f)_{\text {upper } \mid 0}\right]^{2} \mathrm{~d} f=\frac{P_{r}^{2}}{L \Delta f}\left[k+\frac{k(k-1)}{p}\right], \\
\int_{0}^{\infty}\left[S(f)_{\text {lower }}\right]^{2} \mathrm{~d} f=\frac{P_{r}^{2}}{L p^{2} \Delta f}\left[k p+k(k-1)\left(1-\frac{1}{p}\right)\right] .
\end{gathered}
$$

Finally, we may calculate $\tau_{c \text {,upper|b}}$ and $\tau_{c, \text { lower }}$ using Eq. (16), and then substitute into Eq. (12) to reach the desired variances:

$$
\sigma_{c, \text { upper } \mid 1}^{2}=\frac{B P_{r}^{2}}{2 L \Delta f}\left[p+3 k+1+\frac{k(k-1)}{p}\right],
$$

$$
\sigma_{c, \text { upper } \mid 0}^{2}=\frac{B P_{r}^{2} k}{2 L \Delta f}\left[1+\frac{k-1}{p}\right]
$$

$$
\sigma_{c, \text { lower }}^{2}=\frac{B P_{r}^{2} k}{2 L p^{2} \Delta f}\left[p+(k-1)\left(1-\frac{1}{p}\right)\right] .
$$

It should be pointed out the previous analysis is overlapping with the work presented in $[\underline{14}, \underline{15}]$ to some extent. However, this is unavoidable to prevent possible ambiguity.

2) BER Calculations: In [24], a brief description of the incoherent case, in which the integration time is much longer than the coherence time, is presented. It has been stated that the relatively long bit duration causes many statistically independent fluctuations of the instantaneous intensity to occur within the interval $T$. Thus, using the central limit theorem, the noise PDF may be assumed to 
be asymptotically normal. In fact, a truncated Gaussian must be involved in the analysis to avoid the tails of the Gaussian distribution. However, for the sake of the mathematical simplification, we can neglect the negative part of the PDF. This assumption seems to be decent, especially for large mean values, which is the case when the number of active users increases. It is also noteworthy that the area between (mean $\pm 3 \sigma$ ) represents $\cong 98 \%$ of the PDF total area. Hence, applying this argument to our case, we find that the neglected part will not exceed $2 \%$ of the total area. Gaussian approximation has been also assumed in [14].

Finally, we can start the calculation procedure of the BER that results from the PIIN. As mentioned earlier, this effect appears not only in the decision variable $P_{\text {upper }}$, but also in the variable threshold power level $P_{\text {th }}$ as it is a function of $P_{\text {lower. }}$. Hence, in order to more precisely evaluate the optical layer performance, we calculate a new probability of error in each core node referred to as the MAI canceler probability of error and labeled the per node $\mathrm{BER}_{\mathrm{MAI}}$.

First, we obtain the PDF of $P_{X}(x)$ of the decision variable $X$, which is in this case the output power $P_{\text {upper }}$ of FBG1. Then, we get the $\operatorname{PDF} P_{Y}(y)$ of the random variable $Y$ representing the threshold power level $P_{\text {th }}$ :

$$
P_{X \mid b}(x)=\frac{e^{\frac{-\left(x-x_{0 \mid b}\right)^{2}}{2 \sigma_{x \mid b}^{2}}}}{\sqrt{2 \pi \sigma_{x \mid b}^{2}}},
$$

where $b \in\{0,1\}$,

$$
\begin{aligned}
& x_{o \mid 0}=\frac{P_{r}}{L} k, \quad \text { for } \quad b=0, \\
& x_{o \mid 1}=\frac{P_{r}}{L}(p+k+1), \quad \text { for } \quad b=1,
\end{aligned}
$$

and $\sigma_{x \mid b}^{2}$ is given by Eqs. (26) and (27). On the other hand

$$
P_{Y}(y)=\frac{e^{\frac{-\left(y-y_{0}\right)^{2}}{2 \sigma_{y}^{2}}}}{\sqrt{2 \pi \sigma_{y}^{2}}} .
$$

It is not the optimum value, but for simplicity we use a midway threshold. As seen in Fig. $2, P_{\text {bias }}$ is generated in the device to adjust the threshold power in the midway [9]. In our analysis, for simplicity, we assume that $P_{\text {bias }}$ is deterministic and its value is uniform over $\Delta \nu$ :

$$
y_{o}=\frac{P_{r}}{2 L}(p+2 k+1),
$$

and $\sigma_{y}^{2}$ is given by Eq. (28).

Now, we evaluate the $\mathrm{BER}_{\mathrm{MAI}}$ :

$$
\mathrm{BER}_{\mathrm{MAI}}=\frac{1}{2} P_{e \mid 0}+\frac{1}{2} P_{e \mid 1}
$$

Taking into account that $P_{\text {upper }}, P_{\text {th }}$, and $k$ are random variables, we deduce that

$$
\begin{aligned}
\operatorname{BER}_{\mathrm{MAI}}= & \frac{1}{2} \sum_{k=0}^{K} \int_{-\infty}^{\infty} \int_{y}^{\infty} P_{X \mid 0}(x) \cdot P_{Y}(y) \cdot P_{K}(k) \mathrm{d} x \mathrm{~d} y \\
& +\frac{1}{2} \sum_{k=0}^{K} \int_{-\infty}^{\infty} \int_{-\infty}^{y} P_{X \mid 1}(x) \cdot P_{Y}(y) \cdot P_{K}(k) \mathrm{d} x \mathrm{~d} y,
\end{aligned}
$$

where $P_{K}(k)$ is the binomial distribution of the random variable $k$, representing the probability that the number of interferers sending " 1 " is $k$ out of $K$ active users, and $K \in\left\{0,1,2, \ldots, p^{2}-1\right\}:$

$$
\begin{aligned}
\operatorname{BER}_{\mathrm{MAI}}= & \left(\frac{1}{2}\right)^{K+1} \sum_{k=0}^{K}\left(\begin{array}{c}
K \\
k
\end{array}\right) \\
& \times\left[\int_{-\infty}^{\infty} \int_{y}^{\infty} \frac{e^{\frac{-\left(x-x_{0}\right)^{2}}{2 \sigma_{x \mid 0}^{2}}}}{\sigma_{x \mid 0} \sqrt{2 \pi}} \cdot \frac{e^{\frac{-\left(\left(y-y_{0}\right)^{2}\right.}{2 \sigma_{y}^{2}}}}{\sigma_{y} \sqrt{2 \pi}} \mathrm{d} x \mathrm{~d} y\right] \\
& +\left(\frac{1}{2}\right)^{K+1} \sum_{k=0}^{K}\left(\begin{array}{c}
K \\
k
\end{array}\right) \\
& \times\left[\int_{-\infty}^{\infty} \int_{-\infty}^{y} \frac{e^{\frac{-\left(x-x_{o \mid 1}\right)^{2}}{2 \sigma_{x \mid 1}^{2}}}}{\sigma_{x \mid 1} \sqrt{2 \pi}} \cdot \frac{e^{\frac{-\left(y-y_{0}\right)^{2}}{2 \sigma_{y}^{2}}}}{\sigma_{y} \sqrt{2 \pi}} \mathrm{d} x \mathrm{~d} y\right],
\end{aligned}
$$

$$
\begin{aligned}
\operatorname{BER}_{\mathrm{MAI}}= & \left(\frac{1}{2}\right)^{K+2} \sum_{k=0}^{K}\left(\begin{array}{c}
K \\
k
\end{array}\right) \\
& \times \int_{-\infty}^{\infty} \frac{e^{\frac{-\left(y-y_{0}\right)^{2}}{2 \sigma_{y}^{2}}}}{\sigma_{y} \sqrt{2 \pi}} \cdot \operatorname{erfc}\left(\frac{y-x_{o \mid 0}}{\sigma_{x_{o \mid 0} \sqrt{2}}}\right) \mathrm{d} y \\
& +\left(\frac{1}{2}\right)^{K+2} \sum_{k=0}^{K}\left(\begin{array}{l}
K \\
k
\end{array}\right) \\
& \times \int_{-\infty}^{\infty} \frac{e^{\frac{-\left(y-y_{0}\right)^{2}}{2 \sigma_{y}^{2}}}}{\sigma_{y} \sqrt{2 \pi}} \cdot \operatorname{erfc}\left(\frac{x_{o \mid 1}-y}{\sigma_{x \mid 1} \sqrt{2}}\right) \mathrm{d} y .
\end{aligned}
$$

It can be shown that

$$
\int_{-\infty}^{\infty} e^{-(\alpha x+\beta)^{2}} \operatorname{erfc}(\gamma x+\delta) \mathrm{d} x=\left[\frac{\sqrt{\pi}}{\alpha} \operatorname{erfc}\left(\frac{\alpha \delta-\beta \gamma}{\sqrt{\alpha^{2}+\gamma^{2}}}\right)\right] .
$$

Neglecting the negative part of the PDF as stated earlier, the two integrals in the first and second terms of Eq. (34) can be put in the precedent form with different values of $\alpha, \beta, \gamma$, and $\delta$. Thus, after some simple manipulations, Eq. (34) reduces to

$$
\begin{aligned}
\operatorname{BER}_{\mathrm{MAI}}= & \left(\frac{1}{2}\right)^{K+2} \sum_{k=0}^{K}\left(\begin{array}{c}
K \\
k
\end{array}\right) \cdot \operatorname{erfc}\left[\frac{-x_{o \mid 0}+y_{o}}{\sqrt{2\left(\sigma_{x \mid 0}^{2}+\sigma_{y}^{2}\right)}}\right] \\
& +\left(\frac{1}{2}\right)^{K+2} \sum_{k=0}^{K}\left(\begin{array}{c}
K \\
k
\end{array}\right) \cdot \operatorname{erfc}\left[\frac{x_{o \mid 1}+y_{o}}{\sqrt{2\left(\sigma_{x \mid 1}^{2}+\sigma_{y}^{2}\right)}}\right]
\end{aligned}
$$


Now, we substitute back for $x_{o \mid 0}, \sigma_{x \mid 0}, x_{o \mid 1}, \sigma_{x \mid 1}, y_{o}$, and $\sigma_{y}$ to get the $\mathrm{BER}_{\mathrm{MAI}}$ as a function of $k$ :

$$
\begin{aligned}
\operatorname{BER}_{\mathrm{MAI}}= & \left(\frac{1}{2}\right)^{K+2} \sum_{k=0}^{K}\left(\begin{array}{l}
K \\
k
\end{array}\right) \\
& \times\left[\operatorname{erfc}\left(\frac{\Delta f(p+1)}{M}\right)^{\frac{1}{2}}+\operatorname{erfc}\left(\frac{\Delta f(3 p+4 k+3)^{2}}{Q}\right)^{\frac{1}{2}}\right],
\end{aligned}
$$

where

$$
M=4 B k\left[p+k+\frac{(k-1)}{p^{2}}(p-1)\right]
$$

and

$$
Q=4 B L\left[p+3 k+1+\frac{k^{2}}{p}+\frac{k(k-1)}{p^{3}}(p-1)\right] .
$$

Finally, it should be noted that the treatment presented in the previous analysis is similar to that of a balanced detector because, as stated earlier, functions of both devices are analogous. Nevertheless, this treatment is not exactly the same as a balanced detector because there exist two main differences between the two devices. First, the threshold of the optical hard limiter is controllable. In other words, the device is tuned to the desired threshold using the power level reflected from FBG1 (which is related to the power level of the interfering signals). This has been formulated in the mathematical model by introducing the random variable $Y$ and applying the total probability theorem as can be seen in Eqs. (30) and (32), respectively. Next, the balanced detector converts the signal from the optical domain to the electronic domain, which implies taking the shot noise as well as other factors into account.

\section{Numerical Results}

Our results are obtained under the following assumptions: a bit rate $R_{b}=40 \mathrm{~Gb} / \mathrm{s}, \Delta \lambda=40 \mathrm{~nm}$, and the average burst length $=1$ Mbit. First, employing Eqs. (1), (י), and (5), the BL rate is portrayed in Figs. 3 and 4 versus the offered load $\rho$ for different values of $K$. The curves reveal that the effect of the MAC layer restriction is obvious for small values of $K$. However, this effect disappears rapidly as $K$ increases. The reason for this behavior is that we assumed that the offered load is uniformly distributed over the available number of resources $N$. This reduces the effect of increasing the offered load on each resource. Hence, only strong restriction on the allowable number of users (when $K$ is small) affects the system performance.

Next, assuming an ideal OBS/WDM physical layer we perform a comparison between OBS/WDM and OBS/ SAC-OCMDA using the BELR in Fig. 5. First, we use Eq. (36) in Eq. (10) to get the SAC-OCDMA BurstER; then we employ Eqs. (3), (10), and (11) to plot the BELR versus the offered load for both systems in Fig. 5 . In this evaluation, it has been assumed that $l_{e}=20$, and the number

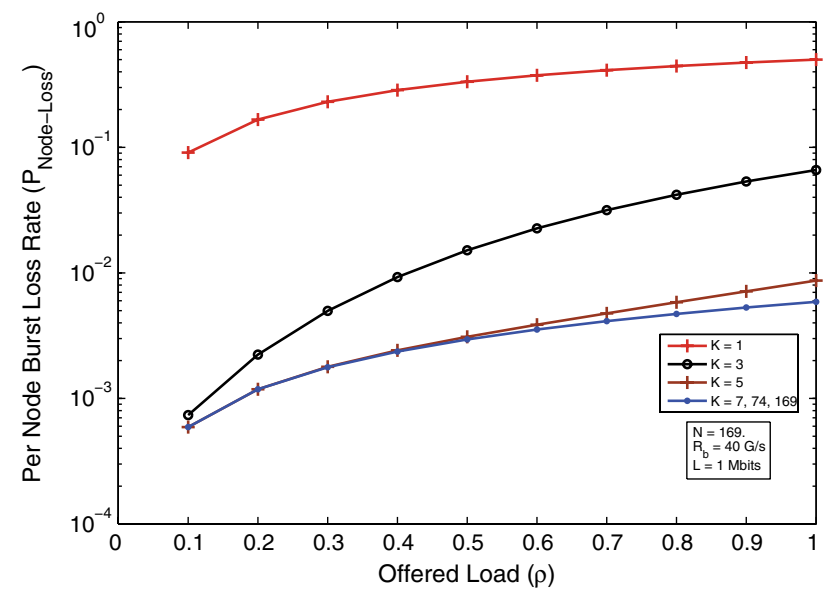

Fig. 3. BL rate versus the offered load $\rho$ for SAC-OCDMA ideal and practical cases. The overall MAI canceler BER can be found as in Eq. ( $)$.

of hops $H=1$. Moreover, for simplicity, we have assumed the same arrival rate for both OBS/WDM and OBS/SACOCMDA. The SAC-OCDMA curves represent the cases of $p=13$ and 17 corresponding to $\delta \lambda=0.2366 \mathrm{~nm}$ and $0.01307 \mathrm{~nm}$, respectively, where $\delta \lambda$ is the grating tunable linewidth. Observing the curves, we find that the WDM system performance, as measured by the BELR parameter, is better for low traffic load because, in this case, the physical layer effect is much stronger. In other words, the MAI canceler BER damps the SAC-OCDMA system performance. As the load increases, the burst blocking becomes more likely, and the need for higher system capacity becomes more evident. That is, the MAC layer performance of OBS/SAC-OCDMA is better than that of OBS/WDM, as has been proved in [9]. Hence, as can be seen in Fig. 5, the SAC-OCDMA MAC layer capabilities appear strongly when the offered load increases.

In Fig. 6, Eq. (5) is used to consider the case of PC assuming that $l_{e}=30$. Clearly, the intervention of resource converters strongly boosts the performance of both systems. Furthermore, the first curve $(C=1)$ shows that the

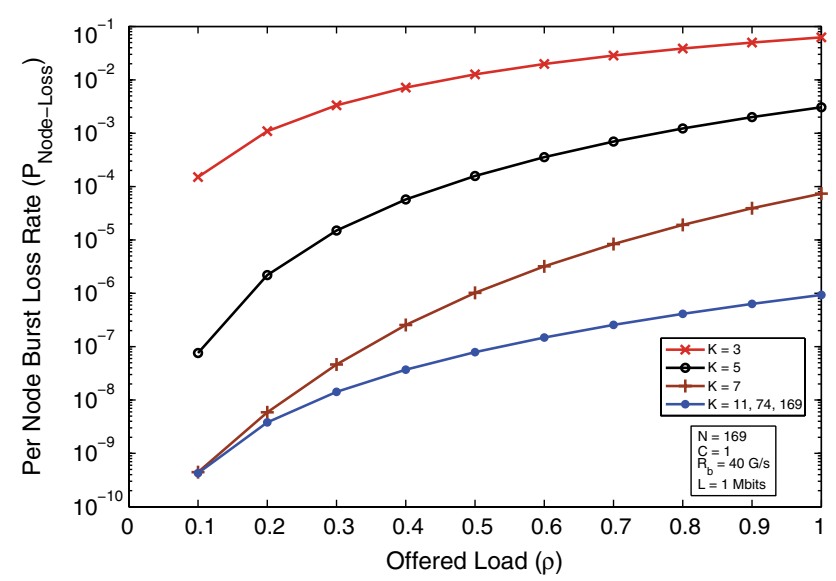

Fig. 4. BL rate versus the offered load $\rho$ for SAC-OCDMA ideal and practical cases in the presence of code converters, $C$. 


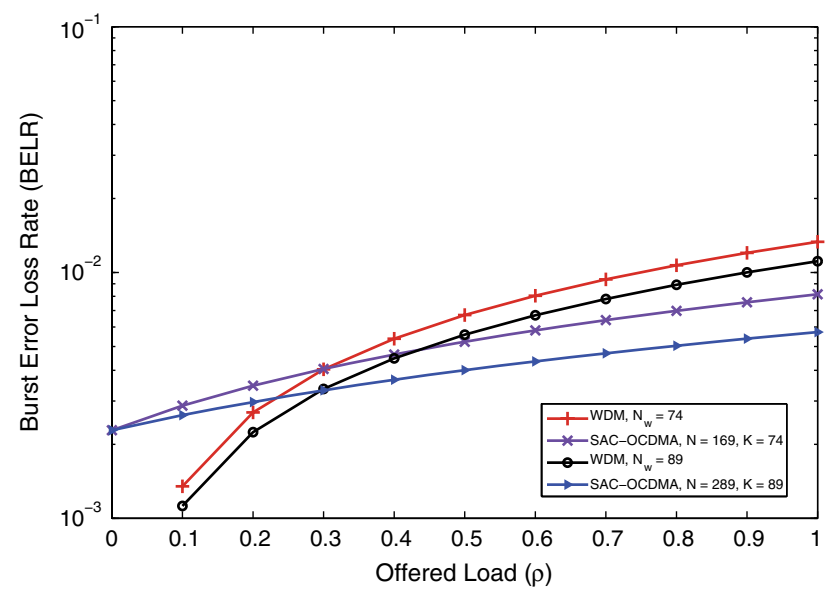

Fig. 5. BELR versus the offered load $\rho$ for WDM and SAC-OCDMA under different values of $K$.

MAC layer performance is always dominant, even for low traffic. However, observing the second curve $(C=2)$, we deduce that the physical layer effect in the OBS/SACOCDMA case reappears for low traffic. In other words, in such a case the effect of the OBS/SAC-OCDMA physical layer is stronger than the enhancement in the MAC layer performance.

In fact, as can be deduced from Figs. 7 and 8, the system behavior portrayed in Figs. 5 and 6 depends on $l_{e}$. Hence, we illustrate in Fig. 7 the relationship between the BELR and the number of tolerated bits in error per burst $l_{e}$ assuming that the offered load $\rho=0.5$. The figure reveals that the OBS/SAC-OCDMA performance is better when $l_{e}$ exceeds a fixed value for each $p$. We also note that when $l_{e}$ exceeds this value the BELR remains constant because $l_{e}$ increases. That is, the BELR consists of two parts: BL probability, which does not depend on $l_{e}$, and BurstER, which depends on $l_{e}$. When this dependency is negligible, the overall behavior remains constant although the BurstER may be the dominant part. In other words, the MAC layer capabilities represent a prevailing factor in the comparison

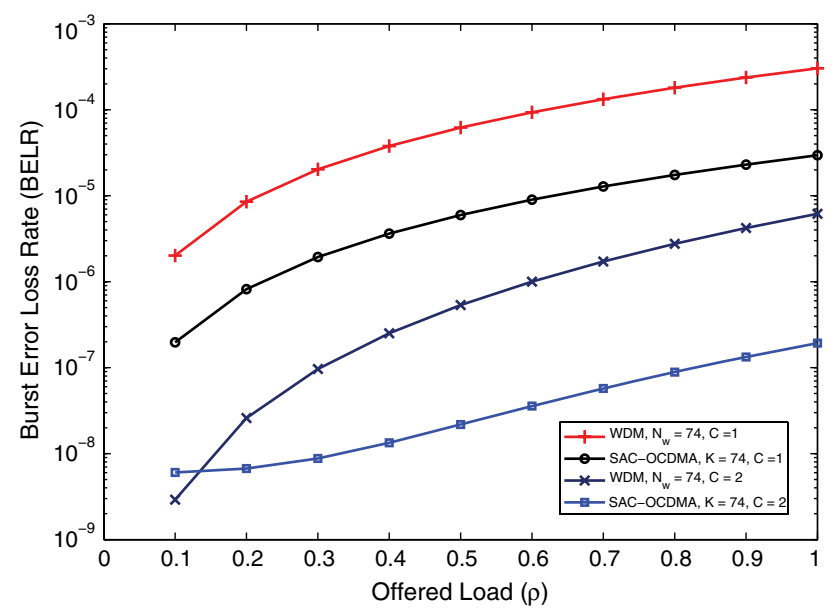

Fig. 6. BELR versus the offered load $\rho$ for WDM and SAC-OCDMA under different values of $K$ and $C$.

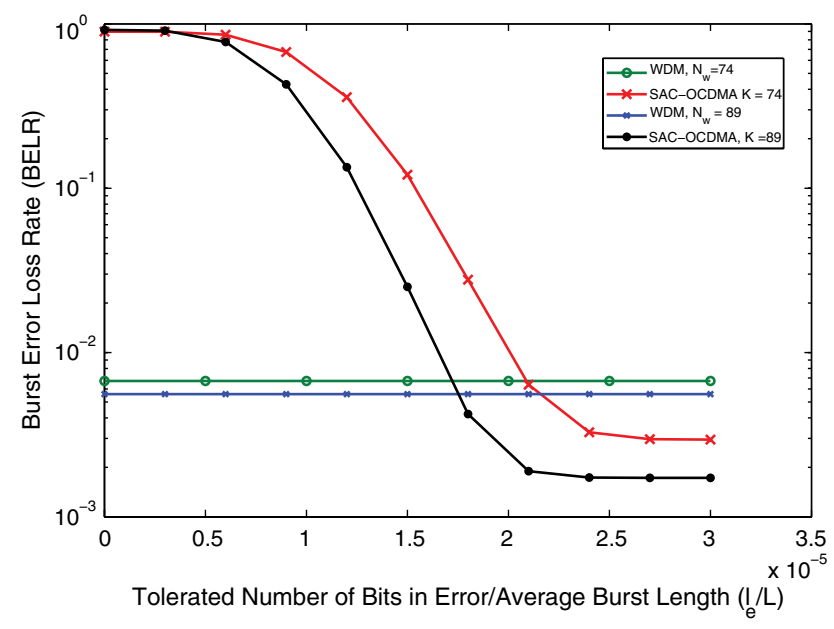

Fig. 7. BELR versus the ratio of the number of tolerated bits in error per burst to the burst length, $l_{e} / L$.

as $l_{e}$ increases. On the other hand, Fig. 8 shows that in the case of code converters, the OBS/SAC-OCDMA system BELR outperforms that of OBS/SAC-OCDMA at a higher value of $l_{e}$. That is, as stated earlier, the presence of code converters enhances the MAC layer performance of both systems. However, due to the noisy physical layer in OBS/SAC-OCDMA, this enhancement in such a case is not strong enough. Hence, a higher value of $l_{e}$ is required.

Note that the BELR is flat against the $l_{e}$ for OBS/WDM because an ideal OBS/WDM physical layer is assumed, which is not the case in a practical network. In fact, in practice both SAC-OCDMA and WDM physical layers can suffer from many types of linear and nonlinear impairments. However, all these effects manifest themselves in terms of the BER. Thus, the presented model, in which the BER is embedded into the BurstER, can be effectively used in developing the network optimum design.

Moreover, it should be pointed out from Fig. 5 that the decrease in $\delta \lambda$ increases $p$ and consequently the number of

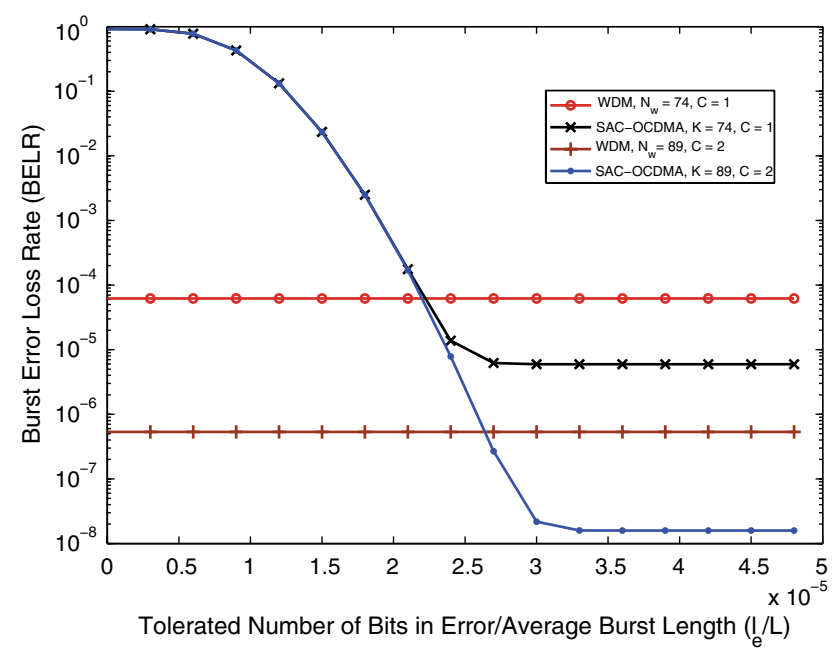

Fig. 8. BELR versus the ratio of the number of tolerated bits in error per burst to the burst length, $l_{e} / L$, for different values of $C$. 


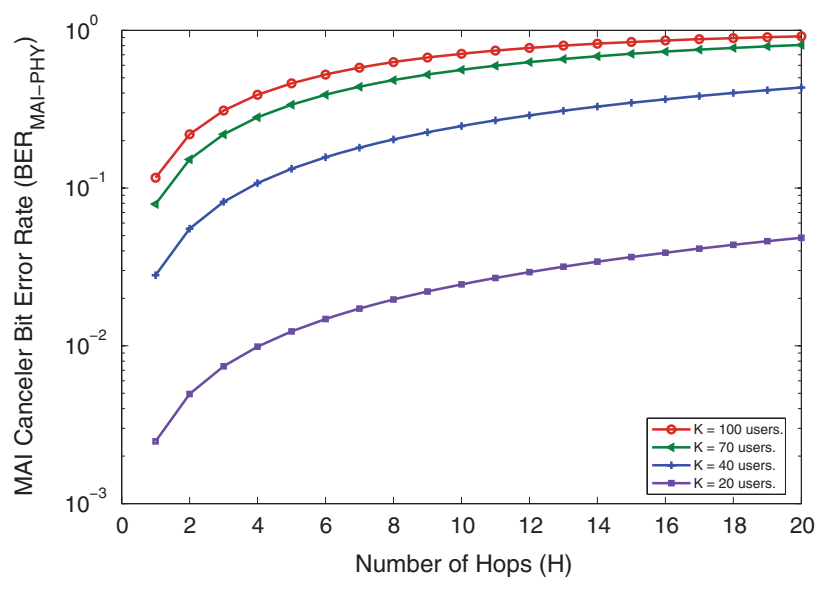

Fig. 9. Probability of bit error versus the number of hops $H$ for different values of $K$.

available codes significantly for the same bandwidth and the same bit rate [14]. Hence, the improvement in the system performance is remarkable, which is not the case for the WDM, where the number of available channels is limited by the bit rate. Nevertheless, we notice that the decrease in $\delta \lambda$ is restricted by the FBG technology.

Finally, the relation between the BER and the number of hops is portrayed in Fig. 9. Observing the figure, we deduce that the BER increases with the number of hops. The reason behind this behavior is that the MAI canceler, which is the source of bit error, is required in every core node to perform a code-based switching operation. Furthermore, it should also be noted that the system behavior deteriorates when the number of interfering users $K$ increases. This illustrates the PIIN effect in the MAI canceler, which generates a BER that depends on the number of interfering users.

\section{ConClusion}

In this paper, a detailed evaluation approach of OBS systems has been proposed. This approach is based on introducing a new measure that combines both MAC and optical layer characteristics. Next, the effect of the PIIN on the OBS/SAC-OCDMA system capacity is presented. The aim of this analysis is to find a closed form of the MAI canceler BER in terms of the number of simultaneous active users, which represents a main factor in the comparison between OBS/SAC-OCDMA and OBS/WDM systems.

Using the presented approach and the found BER formula, we have conducted a performance comparison between OBS/SAC-OCDMA and OBS/WDM systems. It has been shown that the OBS/SAC-OCDMA system outperforms the OBS/WDM system when the number of tolerated bits in error per burst exceeds a fixed value for each $p$, assuming the same spectral width.

Briefly, the main purpose of the paper is to present an assessment model that would be useful in evaluating the performance of both systems, OBS/SAC-OCDMA and OBS/WDM. Next, in order to illustrate the developed model in a clear manner, we employed a simplified example that assumed an ideal WDM physical layer, which is not entirely accurate. In this example we aimed at two main issues:

- To focus on and support the paper's main ideas without confusing the reader. Therefore, we assumed an ideal WDM physical layer and assumed that the SAC-OCDMA suffers from PIIN only.

- To give an illustrative example that employs the proposed evaluation approach rather than to favor one system over the other. For these reasons, some factors have not been taken into account for the sake of simplicity.

Moreover, in the previous results a data rate of $40 \mathrm{~Gb} / \mathrm{s}$ was used. In fact, it remains a questionable issue whether a SAC/OCDMA system that employs an ASE source can reach such a high data rate or not. However, as described in [9], the system employs external modulation. Hence, the maximum achievable data rate depends on the used switch and not on the ASE source.

Other important factors that impact the system data rate and latency include the following: the tunability speed of the FBGs, which depends on its sensitivity to variation in temperature and strain and is hence governed by the fabrication technology. In addition, the availability of a high-speed variable threshold optical hard limiter.

\section{FUTURE WORK}

Indeed, in order to reach an accurate network design, ignored effects such as linear and nonlinear impairments that could affect the physical layers of both systems must be taken into consideration. Such effects are substantially subject to network conditions. Thus, an extensive case study should be conducted in order to reach an optimum network design.

For example, beat noise exists in spectrum-sliced (SS) WDM, which is proposed to replace costly and complex stable laser sources [25]. Moreover, the number of tolerated bits in error $l_{e}$ represents an essential parameter in the model. Therefore, in this case study, the employed burst aggregation protocols, the error detection and correction techniques, the adopted WDM source, and the destination receiver noise must be taken into consideration. This would allow the designer to figure out the exact value of $l_{e}$ and hence to determine using our model whether to use WDM or SAC-OCDMA techniques. Finally, the realistic network topology (with practical distances between hops) would be involved, and the accompanying offered load values as well as the resulting delay could be considered. In this sense, the control logic in the case of code conversion would also be specified.

\section{REFERENCES}

[1] C. Qiao and M. Yoo, "Optical burst switching (OBS)—A new paradigm for an optical Internet," J. High Speed Netw., vol. 8, no. 1, pp. 69-84, Jan. 1999. 
[2] T. Battestilli and H. Perros, "An introduction to optical burst switching," IEEE Commun. Mag., vol. 41, no. 8, pp. S10-S15, Aug. 2003.

[3] X. Yu, J. Li, X. Cao, Y. Chen, and C. Qiao, "Traffic statistics and performance evaluation in optical burst switched networks," J. Lightwave Technol., vol. 22, no. 12, pp. 27222738, Dec. 2004.

[4] X. Yu, Y. Chen, and C. Qiao, "Study of traffic statistics of assembled burst traffic in optical burst switched networks," Proc. SPIE, vol. 4874, pp. 149-159, 2002.

[5] I. Baldine, G. N. Rouskas, H. G. Perros, and D. Stevenson, "Jump-start: A just-in-time signaling architecture for WDM burst-switched networks," IEEE Commun. Mag., vol. 40, no. 2, pp. 82-89, Feb. 2002.

[6] J. Y. Wei and J. R. I. McFarland, "Just-in-time signaling for WDM optical burst switching networks," J. Lightwave Technol., vol. 18, no. 12, pp. 2019-2037, Dec. 2000.

[7] A. I. A. El-Rahman, S. I. Rabia, and H. M. H. Shalaby, "MAC layer performance enhancement using control packet buffering in optical burst switched networks," J. Lightwave Technol., vol. 30, no. 11, pp. 1578-1586, June 2012.

[8] K. Kamakura, O. Kabranov, D. Makrakis, and I. Sasase, "OBS networks using optical code division multiple access techniques," in IEEE Int. Conf. on Communications (ICC), Paris, France, June 2004, pp. 1725-1729.

[9] M. Y. S. Sowailem, M. H. S. Morsy, and H. M. H. Shalaby, "Employing code domain for contention resolution in optical burst switched networks with detailed performance analysis," $J$. Lightwave Technol., vol. 27, no. 23, pp. 5284-5294, Dec. 2009.

[10] B. Moslehi, "Noise power spectra of optical two-beam interferometers induced by the laser phase noise," J. Lightwave Technol., vol. 4, no. 11, pp. 1704-1710, Nov. 1986.

[11] M. M. Rad and J. A. Salehi, "Phase-induced intensity noise in digital incoherent all-optical tapped-delay line systems," J. Lightwave Technol., vol. 24, no. 8, pp. 3059-3072, Aug. 2006.

[12] M. Arie and M. Tur, "Phase-induced intensity noise in optical interferometers excited by semiconductor lasers with nonLorentzian lineshapes," J. Lightwave Technol., vol. 8, no. 1, pp. 1-6, Jan. 1990.

[13] E. D. J. Smith, R. J. Blaikie, and D. P. Taylor, "Performance enhancement of spectral-amplitude-coding optical CDMA using pulse-position modulation," IEEE Trans. Commun., vol. 46, no. 9, pp. 1176-1185, Sept. 1998.

[14] Z. Wei, H. M. H. Shalaby, and H. Ghafouri-Shiraz, "Modified quadratic congruence codes for fiber Bragg-grating-based spectral-amplitude-coding optical CDMA systems," J. Lightwave Technol., vol. 19, no. 9, pp. 1274-1281, Sept. 2001.

[15] Z. Wei, H. M. H. Shalaby, and H. Ghafouri-Shiraz, "New code families for fiber-Bragg-grating-based spectral-amplitudecoding optical CDMA systems," IEEE Photon. Technol. Lett., vol. 13, no. 8, pp. 890-892, Aug. 2001.

[16] M. Izal, J. Aracil, D. Morat, and E. Magaa, "Delay-throughput curves for timer-based OBS burstifiers with light load," J. Lightwave Technol., vol. 24, no. 1, pp. 277-285, Jan. 2006.

[17] D. Gross and C. M. Harris, Fundamentals of Queueing Theory, 3rd ed. Wiley, 1998.

[18] N. Akar, E. Karasan, and K. Dogan, "Wavelength converter sharing in asynchronous optical packet/burst switching: An exact blocking analysis for Markovian arrivals," IEEE J. Sel. Areas Commun., vol. 24, no. 12, pp. 69-80, Dec. 2006.

[19] A. Papoulis and S. U. Pillai, Probability, Random Variables and Stochastic Processes. McGraw-Hill, 2002.
[20] L. Kleinrock, Queueing Systems, vol. 1. Wiley, 1975.

[21] R. A. Griffin, D. D. Sampson, and D. A. Jackson, "Coherence coding for photonic code-division multiple access networks," J. Lightwave Technol., vol. 13, no. 9, pp. 1826-1837, Sept. 1995.

[22] K. W. Chu and F. M. Dickey, "Optical coherence multiplexing for interprocessor communications," Opt. Eng., vol. 30, no. 3, pp. 337-344, May 1991.

[23] X. Zhou, H. M. H. Shalaby, and C. Lu, "Design and performance analysis of a new code for spectral amplitude coding optical CDMA systems," in Proc. IEEE Sixth Int. Symp. on Spread Spectrum Techniques and Applications (ISSSTA), Sept. 2000, pp. 174-178.

[24] J. W. Goodman, Statistical Optics. New York: Wiley, 1985.

[25] S. B. Sun and M. S. Leeson, "Spectrum-sliced WDM and incoherent optical CDMA: A performance comparison," in Ninth Annu. Postgraduate Symp. on the Convergence of Telecommunications, Networking and Broadcasting, Liverpool, UK, June 2008.

Ahmed I. Abd El-Rahman was born in Alexandria, Egypt, in 1979. He received his B.S. and M.S. degrees from Alexandria University, Egypt, in 2002 and 2012, respectively, all in electrical engineering. He is currently working toward a Ph.D. degree in the Department of Electrical and Computer Engineering, Faculty of Engineering and Applied Science, Queen's University, Kingston, Ontario, Canada. He joined Fardos for investment and constructions as a Project Assistant Manager from 2002 until 2012. In 2013, he joined the Lightwave Research Laboratory at Queen's University as a Research Assistant. His current research interests include DSP for coherent optical communication systems, optical burst switched networks, optical CDMA, and wireless systems. He is a Student Member of the IEEE.

Sherif Rabia received a B.Sc. degree in computer science in 1992 and M.Sc. and Ph.D. degrees in engineering mathematics in 1997 and 2001, respectively, all from Alexandria University, Egypt. Currently, Dr. Rabia is an Associate Professor in the Department of Engineering Mathematics and Physics, Alexandria University, Egypt. His research interest falls in the area of applied probability theory and mainly in queueing theory. This includes analytical, numerical, and algorithmic analysis of queueing models as well as stability and control of such models. His research papers have appeared in the Alexandria Engineering Journal, the Journal of the Korean Statistical Society, Computers and Mathematics with Applications, the International Journal of Applied Mathematics and Statistics, and Transactions on Computational Science.

Hossam M. H. Shalaby (S'83-M'91-SM'99) was born in Giza, Egypt, in 1961. He received his B.S. and M.S. degrees from Alexandria University, Egypt, in 1983 and 1986, respectively, and a Ph.D. degree from the University of Maryland, College Park, Maryland, in 1991, all in electrical engineering. In 1991 he joined the Electrical Engineering Department, Alexandria University, Egypt, and was promoted to the position of Professor in 2001. Currently he is on leave from Alexandria University, where he is a Professor with the Department of Electronics and Communications Engineering, School of Electronics, Communications, and Computer Engineering, Egypt-Japan University of Science and Technology (E-JUST), New Borg El-Arab City, Alexandria, Egypt. From December 2000 to 2004, he was an Adjunct Professor with the Department of Electrical and Information Engineering, Faculty of Sciences and Engineering, Laval University, Quebec, Canada. From September 1996 to February 2001, he was on leave from the Alexandria University, where he was with two of the following places. From September 1996 to January 1998, he was with the 
Electrical and Computer Engineering Department, International Islamic University Malaysia, and from February 1998 to February 2001, he was with the School of Electrical and Electronic Engineering, Nanyang Technological University, Singapore. His research interests include optical communications, optical CDMA, optical burst switching, OFDM technology, and information theory. He received an SRC fellowship from 1987 to 1991 (Systems Research Center, Maryland); State Excellence Award in Engineering Sciences in 2007 (Academy of Scientific Research and Technology, Egypt); Shoman Prize for Young Arab Researchers in 2002 (The
Abdul Hameed Shoman Foundation, Amman, Jordan); State Incentive Award in Engineering Sciences, twice in 1995 and 2001 (Academy of Scientific Research and Technology, Egypt); University Excellence Award in 2009 (Alexandria University, Egypt); and University Incentive Award in 1996 (Alexandria University, Egypt). He has served as a student branch counselor (Alexandria University), IEEE Alexandria and North Delta Subsection, from 2002 to 2006 , and served as a chairman of the student activities committee of the IEEE Alexandria Subsection from 1995 to 1996 . He is a Senior Member of the IEEE. 